\title{
Excursion-volume relation of the right hemidiaphragm measured by ultrasonography and respiratory airflow measurements
}

\author{
Eytan Cohen, Anne Mier, Peter Heywood, Kevin Murphy, Joe Boultbee, \\ Abraham Guz
}

\begin{abstract}
Background - Although real time ultrasonography has been used in the last decade to record diaphragmatic motion, the relation between diaphragmatic excursion and different inspired volumes (VT) has not been assessed by ultrasound. Methods - Ten normal subjects were studied in the supine posture. Diaphragmatic excursion and VT were assessed simultaneously by $M$ mode ultrasonography and respiratory airflow measurements at different inspired volumes. Ultrasound recordings of the movement of the right hemidiaphragm were carried out in the longitudinal plane subcostally. The transducer was held in a fixed position by a frame, built especially to eliminate any artefactual movement caused by outward motion of the anterior abdominal wall on inspiration.
\end{abstract}

Results - Mean (SD) maximal diaphragmatic excursion recorded was $6 \cdot 0$ $(0 \cdot 7) \mathrm{cm}$. Inspired volumes ranged from $15(5) \%$ to $87(10) \%$ of the subjects' inspiratory capacity. A linear relation between diaphragmatic excursion and VT was found in all subjects $(r=0.976-0.995)$. The regression line had a slope of 1.66 $(0 \cdot 24) \mathrm{cm} / 1$. This slope had no correlation with either the height $(r=0 \cdot 007)$ or weight $(r=0 \cdot 143)$ of the subjects. In five subjects in whom diaphragmatic excursion could be recorded at volumes near total lung capacity, the relation between diaphragmatic excursion and VT became alinear at very high lung volumes.

Conclusions - The relation between diaphragmatic excursion and VT was linear between $15(5) \%$ and $87(10) \%$ of inspiratory capacity. Ultrasonography of the diaphragm is a simple technique that could be applied in the clinical investigation of patients with suspected abnormalities of diaphragmatic movement.

(Thorax 1994;49:885-889)

Until the last decade assessment of diaphragmatic movement relied traditionally on fluoroscopic measurements. The exposure to irradiation limits the duration of such studies.
In addition, fixed reference points are difficult to establish because many structures within the field of view move with inspiration. Ultrasonography is a safe and accurate method which is currently used in cardiology with great reliability and reproducibility. This method has recently been used to assess normal movement of the diaphragm in subjects with no respiratory disease $^{12}$ and abnormal movement in patients with diaphragmatic pathology. ${ }^{3}$ The present study assesses the relation between diaphragmatic excursion and different inspired volumes with simultaneous ultrasonography and respiratory airflow measurements. Since inevitable movement of the transducer occurs as a result of abdominal movement during breathing if the probe is held by hand, it was necessary to build a special device to hold the transducer in a fixed position.

\section{Methods}

SUBJECTS

Studies were performed on 10 healthy subjects (five men) with no history of respiratory disease. Their age was 31 (6) years, height $170(9) \mathrm{cm}$, and weight 67 (11) kg. Nine of the subjects were either physiologists or technicians from the department of respiratory medicine and one was a naive subject. All gave their verbal informed consent to the study.

\section{LUNG FUNCTION STUDIES}

Volume and flow measurements were performed in the lung function laboratory. Volume measurements were carried out in the supine posture using the helium dilution technique. Flow measurements were performed in the seated posture.

\section{DIAPHRAGMATIC EXCURSION}

Ultrasound records of diaphragmatic excursion were obtained using a Diasonic LR400 with a $3.5 \mathrm{MHz}$ transducer. Measurements were taken in the supine posture in the longitudinal plane. The probe was positioned in the midclavicular line below the right costal margin. After identifying the hemidiaphragm in the B mode the transducer was held using a specially built device which was fixed to the bed and could be adjusted in a variety of angles (fig 1). 


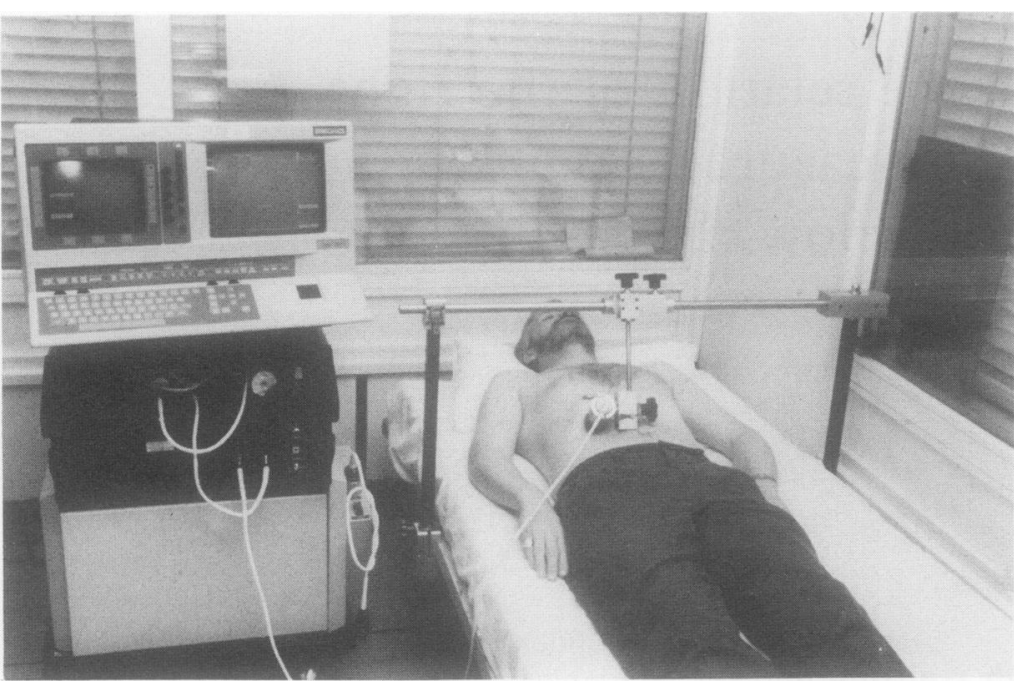

Figure 1 The transducer fixed in position by a specially built frame to display the right hemidiaphragm.

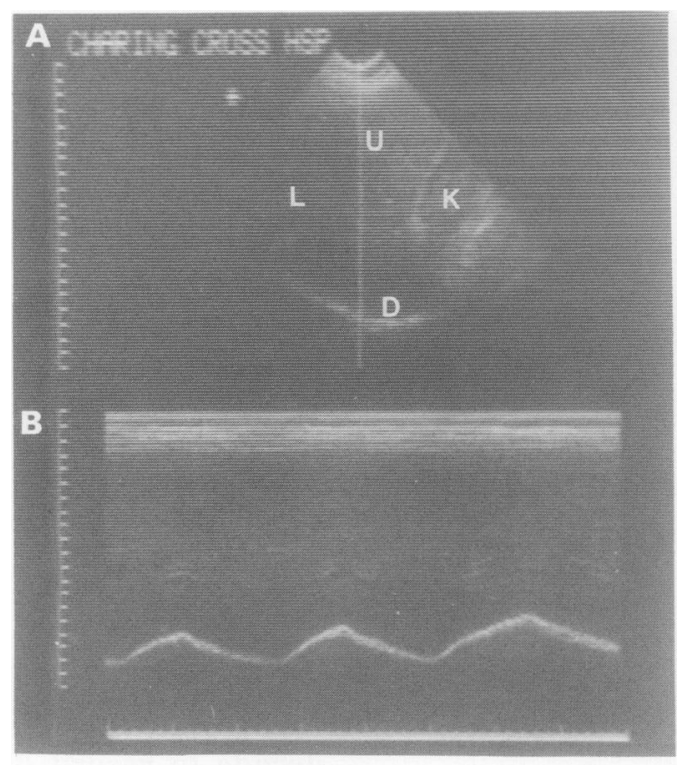

The ultrasound beam was then positioned so that it was approximately parallel to the movement of any part of the hemidiaphragm; this was only found to be possible in relation to the posterior third of the hemidiaphragm during inspiration. Recordings in the $M$ mode at this position were then made throughout the test (figs 2 and 3). Real time scans were recorded on videotape with a Panasonic videocassette recorder (Ag-6200). Using the freeze frame control, each breath was later studied and those selected for measurement were transmitted to photographic film; from this film, excursion was measured using a magnifying glass with a vernier scale which allowed measurements as small as $0.2 \mathrm{~mm}$ to be made with confidence. The measurements were always made from one side of the $M$ mode tracing; haziness at the edge of the signal did not therefore contribute any doubt as to the point from where the measurements should be made. Real measurements of diaphragmatic excursion were finally calculated by using the ultrasound calibration. Depending on the size of the calibration scale selected on the ultrasound machine, diaphragmatic excursion measurements could be made with a sensitivity of $0.5-0.7 \mathrm{~mm}$.

\section{VOLUME MEASUREMENTS}

Subjects wore a noseclip and breathed through a mouthpiece attached to a hot wire respiratory

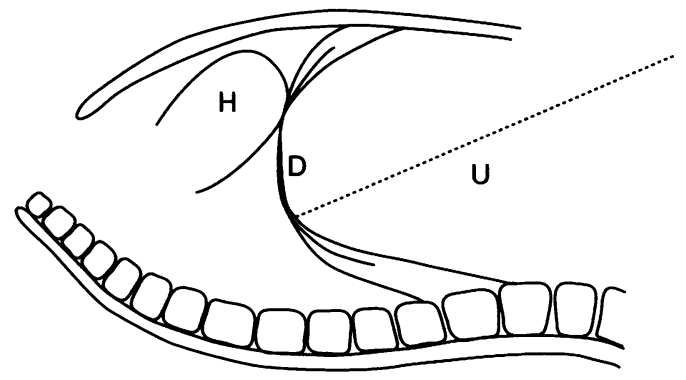

Figure $2 U=$ position of the ultrasound beam for $M$ mode tracing; $D=$ diaphragm; $H=$ heart.

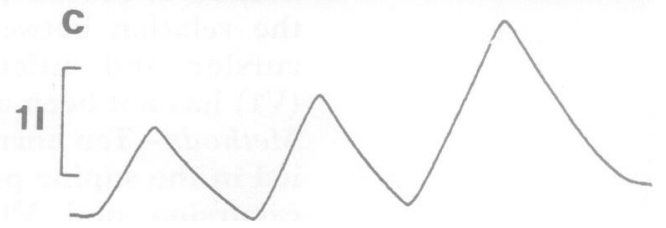

Figure 3 (A) $B$ mode scan: $D=$ diaphragm, $L=$ liver, $K=$ kidney, $U=$ position of the ultrasound beam for $M$ mode tracing which corresponds to the dotted line in fig 2; (B) $M$ mode scan; (C) inspired volume. The ultrasound calibration scale on the vertical axis is in centimetres. The time course on the horizontal axis is 8 seconds. Direction of inspiration in $(A),(B)$, and $(C)$ is upwards.

flowmeter (Minato). The integrated volumes were plotted on a chart recorder (Gould Recorder 2400). Diaphragmatic excursion and volume recordings were synchronised by a simultaneous sound signal on the video tape, and marking on the chart recorder, respectively.

\section{PROTOCOL OF STUDY}

Subjects were instructed to take increasing inspired volumes between resting end expiration and total lung capacity; a visual feedback of their VT could be seen on an oscilloscope positioned in front of them. After a rest of no less than three minutes the procedure was repeated so that at least three adequate runs were recorded. No instructions were given as to the number of breaths to take so that subjects could select their own comfortable increments of increasing inspired volumes, and hence the number of breaths required, to reach total lung capacity. Similarly no instructions were given as to which muscle groups the subjects should use, so that they were free to use the combination of diaphragm and intercostal muscles that they found most comfortable and most normal.

\section{DATA ANALYSIS}

Those breaths during which clear recordings were not obtained on ultrasound were rejected. The run with the maximal number of breaths of different inspired volumes was selected for analysis. Reproducibility between runs was assessed in one subject (subject 4 ) by analysing 
Table 1 Lung function recordings in 10 normal subjects. Volume measurements carried out in supine position

\begin{tabular}{|c|c|c|c|c|c|c|c|c|c|c|c|c|}
\hline \multirow[t]{2}{*}{ Subject } & \multirow[t]{2}{*}{ Sex } & \multirow[t]{2}{*}{ Age (y) } & \multirow[t]{2}{*}{ Height $(\mathrm{cm})$} & \multirow[t]{2}{*}{ Weight (kg) } & \multicolumn{2}{|l|}{$F E V_{1}$} & \multicolumn{2}{|l|}{$F V C$} & \multicolumn{2}{|l|}{$V C$} & \multicolumn{2}{|l|}{$T L C$} \\
\hline & & & & & $l$ & $\%$ pred & $l$ & $\%$ pred & $l$ & $\%$ pred & $l$ & $\%$ pred \\
\hline 1 & $\mathrm{~F}$ & 30 & 180 & 75 & $4 \cdot 27$ & 112 & $5 \cdot 30$ & 113 & $5 \cdot 25$ & 112 & $7 \cdot 30$ & 115 \\
\hline 2 & $\mathrm{~F}$ & 28 & 174 & 81 & 3.75 & 102 & $4 \cdot 67$ & 104 & $4 \cdot 50$ & 100 & $6 \cdot 33$ & 106 \\
\hline 3 & $\mathrm{~F}$ & 23 & 155 & 48 & $2 \cdot 83$ & 89 & $3 \cdot 42$ & 91 & $3 \cdot 38$ & 89 & $4 \cdot 39$ & 96 \\
\hline 4 & $\mathrm{~F}$ & 33 & 165 & 61 & $3 \cdot 48$ & 107 & 4.54 & 113 & $4 \cdot 31$ & 108 & $5 \cdot 86$ & 111 \\
\hline 5 & $F$ & 21 & 163 & 58 & $4 \cdot 31$ & 123 & $4 \cdot 88$ & 116 & $4 \cdot 64$ & 112 & $6 \cdot 11$ & 118 \\
\hline 6 & M & 32 & 183 & 80 & $4 \cdot 81$ & 104 & 5.94 & 102 & 6.25 & 107 & $7 \cdot 60$ & 104 \\
\hline 7 & $\mathbf{M}$ & 43 & 182 & 81 & 3.75 & 88 & $6 \cdot 00$ & 109 & $6 \cdot 13$ & 111 & 8.85 & 117 \\
\hline 9 & $M$ & 28 & 165 & 63 & $3 \cdot 30$ & 102 & 3.96 & 104 & 3.90 & 100 & $4 \cdot 68$ & 88 \\
\hline 10 & $M$ & 31 & 164 & 63 & $3 \cdot 40$ & 113 & $4 \cdot 25$ & 117 & $4 \cdot 08$ & 136 & $5 \cdot 45$ & 110 \\
\hline
\end{tabular}

$\mathrm{FEV}_{1}=$ forced expiratory volume in one second; $\mathrm{FVC}=$ forced vital capacity; $\mathrm{VC}=$ vital capacity; TLC $=$ total lung capacity; $\%$ pred $=\%$ of predicted.

Table 2 Results of diaphragmatic excursion (DE) and tidal volumes (VT) in 10 normal subjects

\begin{tabular}{|c|c|c|c|c|c|c|c|c|c|c|}
\hline \multirow[t]{2}{*}{ Subject } & \multirow{2}{*}{$\underset{(\mathrm{cm})}{\operatorname{Max}} D E$} & \multicolumn{3}{|l|}{$\operatorname{Max} V_{T}$} & \multicolumn{3}{|l|}{$\operatorname{Min} V_{T}$} & \multirow[t]{2}{*}{$r$} & \multirow{2}{*}{$\begin{array}{l}D E / V T \\
(\mathrm{~cm} / \mathrm{l})\end{array}$} & \multirow{2}{*}{$\begin{array}{l}\text { Intercept }(y) \\
(\mathrm{cm})\end{array}$} \\
\hline & & $l$ & $\% I C$ & $\% V C$ & $l$ & $\% I C$ & $\% V C$ & & & \\
\hline 1 & $5 \cdot 7$ & $3 \cdot 5$ & 75 & 78 & $0 \cdot 4$ & 8 & 19 & 0.995 & 1.46 & 0.85 \\
\hline 2 & $7 \cdot 3$ & $3 \cdot 1$ & 89 & 91 & 0.8 & 23 & 40 & 0.983 & $2 \cdot 03$ & 1.04 \\
\hline 3 & $4 \cdot 9$ & $2 \cdot 6$ & 98 & 99 & 0.6 & 23 & 39 & 0.984 & $1 \cdot 75$ & 0.23 \\
\hline 4 & $5 \cdot 8$ & $3 \cdot 6$ & 100 & 100 & 0.4 & 11 & 29 & 0.987 & $1 \cdot 40$ & 1.04 \\
\hline 5 & $5 \cdot 3$ & $3 \cdot 0$ & 83 & 86 & 0.6 & 18 & 34 & 0.976 & 1.44 & 1.28 \\
\hline 6 & $6 \cdot 2$ & 3.9 & 82 & 86 & 0.5 & 11 & 32 & 0.980 & 1.43 & 1.05 \\
\hline 7 & $6 \cdot \overline{8}$ & $3 \cdot 6$ & 76 & 82 & $0 \cdot 6$ & 13 & 33 & 0.992 & 1.58 & 0.64 \\
\hline 8 & $5 \cdot 4$ & $2 \cdot 6$ & 78 & 84 & $0 \cdot 4$ & 12 & 36 & 0.964 & 1.96 & -0.30 \\
\hline 9 & $6 \cdot 0$ & $3 \cdot 0$ & 91 & 92 & 0.4 & 12 & 25 & 0.989 & 1.58 & 1.05 \\
\hline 10 & $6 \cdot 1$ & $3 \cdot 0$ & 100 & 100 & $0 \cdot 4$ & 14 & 41 & 0.978 & 1.74 & 1.23 \\
\hline Mean (SD) & $6.0(0.7)$ & $3 \cdot 2(0 \cdot 4)$ & $87(10)$ & $90(8)$ & $0.5(0 \cdot 1)$ & $15(5)$ & $33(7)$ & $\begin{array}{c}0.983 \\
(0.009)\end{array}$ & $\begin{array}{c}1 \cdot 66 \\
(0 \cdot 24)\end{array}$ & $\begin{array}{c}0.81 \\
(0.49)\end{array}$ \\
\hline
\end{tabular}

$\mathrm{IC}=$ inspiratory capacity; $\mathrm{VC}=$ vital capacity; $r=$ regression coefficient.

two runs on the same day. Between-day reproducibility was assessed in another subject (subject 7) on two separate days.

\section{Results}

Lung function recordings lay within the normal range in all subjects, except subject 8 who was a smoker and whose $\mathrm{FEV}_{1}$ was slightly low (table 1). Owing to the careful design of the frame used to fix the ultrasound probe to the bed, there was no visible movement of the transducer during ultrasound measurements. None of the subjects experienced any difficulty in performing the task, but because of the slight, but definite, pressure of the transducer on the abdomen during recordings, it was noted that they were not all able to achieve their maximal inspiratory capacity (table 2).

Between nine and 17 breaths were analysed for each subject. In most cases all recordings made were suitable for analysis; only one in- spiration was rejected in some of the subjects where a clear recording was not obtained. Results are shown in table 2 . The mean (SD) maximal excursion of the right hemidiaphragm recorded was $6.0(0.7) \mathrm{cm}$. The largest inspiratory volume achieved by the subjects was $87(10) \%$ of their maximal inspiratory capacity and $90(8) \%$ of their relaxed vital capacity. The smallest VT during recording was $15(5) \%$ of their inspiratory capacity and $33(7) \%$ of their vital capacity. A linear correlation (fig 4) was found between diaphragmatic excursion and VT in all subjects $(r=0.964-0.995)$. The regression line had a slope of $1.66(0.24) \mathrm{cm} / 1$ and an intercept $(y)$ of $0.81(0.49) \mathrm{cm}$.

There was no correlation between this slope and either the height $(r=0.007)$ or weight $(r=$ $0 \cdot 143$ ) of the subjects. In the five subjects who were able to achieve inspired volumes close to TLC, the gradient of the relation between diaphragmatic excursion and VT was reduced at high lung volumes (fig 5). Confident meas-
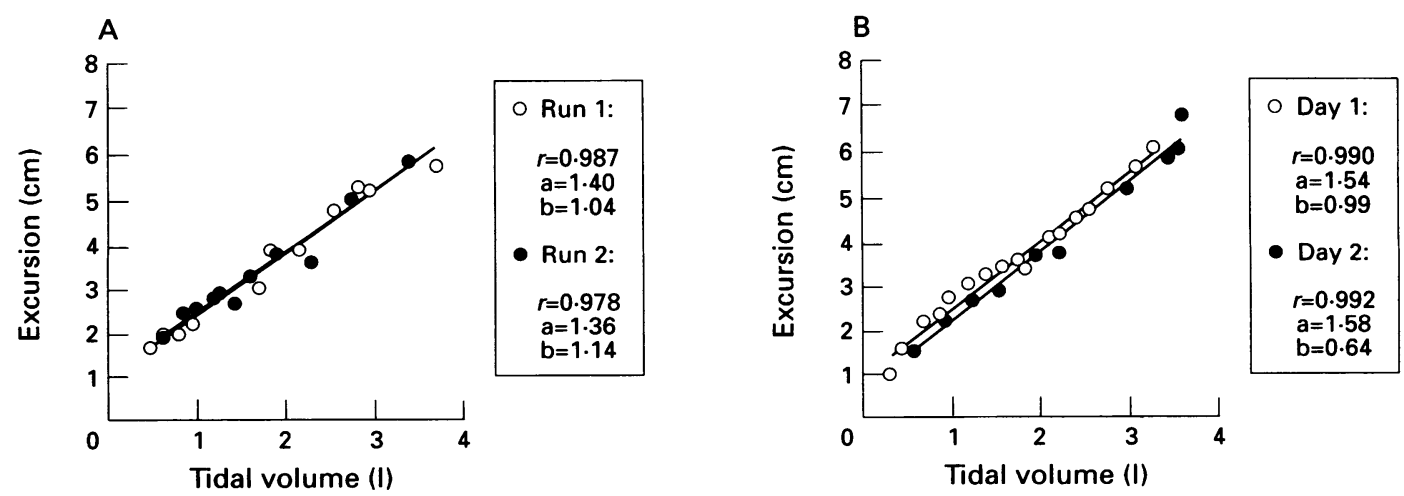

Figure 4 (A) Diaphragmatic excursion-volume relation in subject 4 showing two consecutive runs without repositioning of the transducer. (B) Diaphragmatic excursion-volume relation in subject 7 showing two runs of measurements on two different days. $a=$ slope $(\mathrm{cm} / \mathrm{l}) ; b=$ intercept $(\mathrm{cm})$ on excursion axis. 


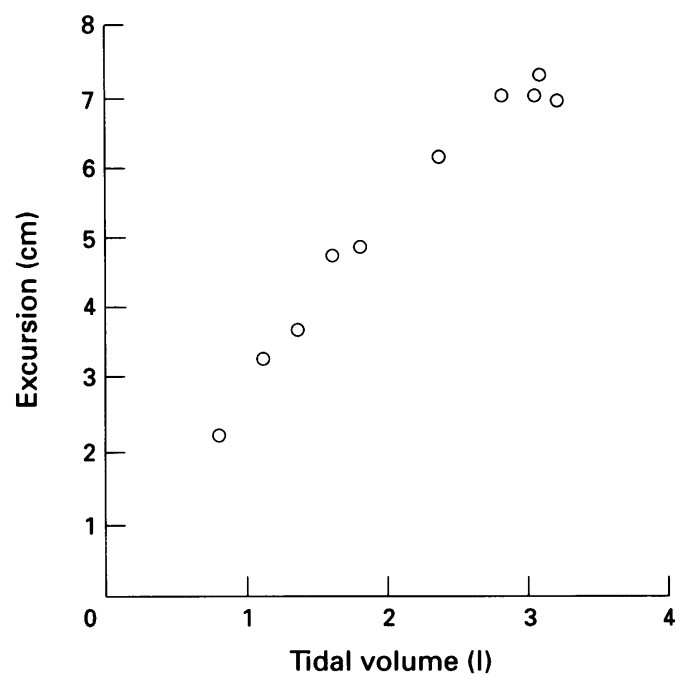

Figure 5 Diaphragmatic excursion-volume relation in subject 2. At lung volumes close to total lung capacity the relation becomes alinear.

urements of $0.5-0.7 \mathrm{~mm}$ diaphragmatic excursion were equivalent to a range of inspired volumes that varied from $25 \mathrm{ml}$ to $49 \mathrm{ml}$ in the various subjects (see appendix). Results between runs and between days were found to be highly reproducible (fig 4 ).

\section{Discussion}

Real time ultrasonography has occasionally been used in the last decade to assess diaphragmatic movement. In one study unequal excursion of the two hemidiaphragms was found in $80 \%$ of 55 patients with no respiratory disease. ${ }^{1}$ In $65 \%$ of these patients there was greater movement of the right side and in $15 \%$ greater movement of the left. In a different study of normal subjects, again using ultrasound, it was shown that the middle and posterior parts of the hemidiaphragm moved more than the anterior part. ${ }^{2}$ This may be explained by the fact that each hemidiaphragm consists of two muscles - a costal part which causes displacement of the anterior portion, and a crural part which displaces the posterior portion. ${ }^{45}$

We aimed to assess the relation between diaphragmatic excursion and VT using simultaneous ultrasonography and airflow measurements. Originally it was planned to measure the diaphragmatic excursion over the full range of vital capacity, but as visualisation of the diaphragm has been found to be difficult on ultrasound at volumes below resting end expiration, measurements were limited to the inspiratory capacity range. We studied only the right hemidiaphragm as the acoustic window through the liver allows clearer pictures to be obtained than on the left. Although the left hemidiaphragm was not assessed, we assume that it behaves similarly to the right.

This is the first study during which the ultrasound transducer has been firmly fixed during recordings of the diaphragm. Although fixing the ultrasound probe by a specially built device may have limited the inspired volumes achieved because of pressure of the transducer on the abdomen, we were confident that we were recording true motion of the posterior part of the diaphragm in the longitudinal plane, with no artifacts due to movement of the probe. The reason for measuring excursion of this part of the hemidiaphragm was to enable us to obtain exact measurements by using the $M$ mode. Only the $M$ mode beam could be directed parallel to the movement when studying the posterior part because of the combined movement of the posterior part of the hemidiaphragm which occurs in a cephalo-caudal axis as well as in a posterior-anterior axis. ${ }^{1} \mathrm{We}$ are unable to say whether similar results would have been obtained if the anterior portion of the diaphragm had been studied. However, since it is impossible to obtain $M$ mode recordings parallel to the movement of the anterior part of the diaphragm, this question cannot be answered.

An early study using fluoroscopy in 10 normal men found that the average excursion of the right hemidiaphragm over the inspiratory capacity range was $7.5 \mathrm{~cm}$ in the supine posture. ${ }^{6}$ In our study excursion of the right hemidiaphragm was $6.0 \quad(0.7) \mathrm{cm}$ at inspired volumes of $87(10) \%$ of maximal inspiratory capacity. These results can be compared with those of previous authors ${ }^{1}$ using ultrasound with a hand-held probe. If the probe is not fixed, movement of the probe almost inevitably occurs as the anterior abdominal wall moves outwards on inspiration. Such movement of the probe would result in falsely low recordings of diaphragmatic excursion because the probe moves outwards with the descent of the diaphragm. The results of Houston et $a^{1}$ who recorded diaphragmatic excursion of 5.3 $(1 \cdot 6) \mathrm{cm}$ at maximal inspiratory capacity probably underestimated the maximal diaphragmatic excursion that occurred.

We have shown an overall linear relation between excursion of the posterior third of the right hemidiaphragm and VT between 15(5)\% and $87(10) \%$ of inspiratory capacity. Since the diaphragm is the main muscle of inspiration, ${ }^{7}$ it was not surprising that the relation between diaphragmatic excursion and VT would be linear in the middle of the vital capacity range. The inspiratory intercostal muscles are also active during inspiration, however, and contribute to rib cage movement and hence to changes in lung volume. Although we did not study the intercostal muscles with ultrasound, it is possible that movement of the intercostals may also correlate with lung volume during inspiration up to about $80 \%$ of inspiratory capacity; this possibility was suggested by our finding that the value of the diaphragmatic excursion (DE)/VT slope varied between subjects and yet the actual relation between diaphragmatic excursion and VT was always linear. Several factors may affect the DE/VT slope; those subjects who used their diaphragm to a greater extent than their intercostals to achieve a certain inspired volume would be expected to have had a higher value of DE/VT and hence a steeper slope. It is known that, compared with men, women often use their intercostal muscles more than their diaphragms on in- 
spiration. It would therefore be expected that DE/VT (and hence the slope) would be less in the women than the men, but we found no such pattern; mean DE/VT was $1.66 \mathrm{~cm} / 1$ in men and $1.62 \mathrm{~cm} / 1$ in women. Although we did not record respiratory inductance plethysmography or electromyograms of the different muscles, our subjects were deliberately not provided with instructions as to which muscle groups to use and we assume that they used different combinations of diaphragm and intercostal muscles to achieve the same lung volumes.

At very high lung volumes, close to maximal inspiratory capacity, one would expect the sternomastoid and scalene muscles to be active. Indeed we found that the relation between diaphragmatic excursion and VT was reduced in the five subjects in whom we could obtain a few recordings at these high volumes (fig 5). This suggested that the accessory muscles were also contributing to inspiration near to maximal inspiratory capacity. Since we calculated the overall DE/VT using these few measurements close to total lung capacity, we may have slightly underestimated DE/VT and hence the slope in the middle of the vital capacity range. Previous studies $^{89}$ also recorded diaphragmatic excursion over the full range of vital capacity using fluoroscopic techniques; these authors similarly found a linear relation between diaphragmatic excursion and VT and noted that this became alinear at volumes close to total lung capacity.

Owing to difficulty in obtaining recordings of the diaphragm by ultrasound at low inspired volumes, we were also unable to measure how much the diaphragm moved just above resting end expiration. Since diaphragmatic excursion must be zero at zero VT (fig 4), it would appear that the DE/VT slope has to be steeper just above resting end expiration. This would mean that for the same increase in lung volume the posterior portion of the diaphragm moves more at the onset of inspiration than later on in inspiration.

The finding that there was no correlation between $\mathrm{DE} / \mathrm{VT}$ and the height or weight of the subjects was not unexpected. Although it would have been interesting to see if there was a correlation between maximum diaphragmatic excursion at total lung capacity and height of the subjects, we were not able to obtain these recordings in all subjects probably because of pressure of the transducer on the abdomen.

In summary, movement of the right hemidiaphragm can be recorded by $M$ mode ultrasonography in the longitudinal plane using a specially built device to fix the probe. Such recordings may be an easy, non-invasive method of assessing movement of the diaphragm in patients with neurological and pulmonary disease.

\section{Appendix}

Derivation of inspired volume sensitivity range from measurements of diaphragmatic excursion (DE):

(1) Measurements of diaphragmatic excursion could be made with a sensitivity of 0.5-0.7 mm (page 886).

(2) Highest value of $\mathrm{DE} / \mathrm{VT}$ recorded = $2.03 \mathrm{~cm} / 1=20.3 \mathrm{~mm} / 1000 \mathrm{ml}$. Most sensitive measurements of $\mathrm{DE}$ of $0.5 \mathrm{~mm}=$ $\frac{0.5 \times 1000}{20.3}=25 \mathrm{ml}$.

(3) Lowest value of $\mathrm{DE} / \mathrm{VT}$ recorded $=$ $1.43 \mathrm{~cm} / 1=14.3 \mathrm{~mm} / 1000 \mathrm{ml}$. Least sensitive measurements of $\mathrm{DE}$ of $0.7 \mathrm{~mm}=$ $\frac{0 \cdot 7 \times 1000}{14 \cdot 3}=49 \mathrm{ml}$.

The authors thank Dr Marc Owen, Consultant Radiologist at West Middlesex Hospital, for his help during the initial stages of the study.

1 Houston JG, Morris AD, Howie CA, Reid JL, McMillan N. Technical report: Quantitative assessment of diaphragmatic
movement - a reproducible method using ultrasound. Clin movement - a reproducib

2 Harris RS, Giovannetti M, Kim BK. Normal ventilatory movement of the right hemidiaphragm studied by ultrasonography and pneumotachography. Radiology 1983;146: $141-4$.

3 Diament MJ, Boechat MI, Kangarloo H. Real-time ultrasound in the evaluation of suspected abnormalities of diaphragmatic motion. $f$ Clin Ultrasound 1985;13:539-43.

4 De Troyer A, Sampson M, Sigrist S, Macklem PT. The diaphragm: two muscles. Science Wash DC 1981;213:237-8.

De Troyer A, Sampson M, Sigrist S, Macklem PT. Action of the costal and crural parts of the diaphragm on the rib cage in dog. $f$ Appl Physiol 1982;53:30-9.

6 Wade OL. Movements of the thoracic cage and diaphragm in respiration. $\mathcal{F}$ Physiol 1954;124:193-212.

7 Goldman M, Mead J. Mechanical interaction between the diaphragm and rib cage. $\mathcal{f}$ Appl Physiol 1973;35:197-204.

Verschakelen JA, Deschepper K, Jiang TX, Demedts M. Diaphragmatic displacement measured by fluoroscopy and derived by respitrace. $\mathcal{F}$ Appl Physiol 1989;67:694-8.

9 Verschakelen JA, Deschepper K, Demedts M. Relationship between axial motion and volume displacement of the diaphragm during VC maneuvers. F Appl Physiol 1992;72: 1536-40. 